\title{
Psychiatric Hospitalizations, Suicides \& At Math
}

Paul T E Cusack

BSc E, DULE, 23 Park Ave. Saint John, NB E2J 1R2, Canada.

Correspondence Author: Paul T E Cusack, BSc E, DULE, 23 Park Ave. Saint John, NB E2J 1R2, Canada.

Received date: January 12, 2021; Accepted date: March 08, 2021; Published date: March 18,2021

Citation: Paul T E Cusack. (2021) Psychiatric Hospitalizations, Suicides \& At Math. Clinical Medical Reviews and Reports 3(3); DOI: $10.31579 / 2690-8794 / 059$

Copyright: (C) 2021 Paul T E Cusack, This is an open access article distributed under the Creative Commons Attribution License, which permits unrestricted use, distribution, and reproduction in any medium, provided the original work is properly cited.

\section{Abstract}

This paper is a brief investigation of the mathematical laws that undergird psychiatric hospitalizations. I wondered with all the mental illness, whether it is increasing over the centuries or staying the same, but perhaps more diagnosed and hospitalized. The answer is that mental illness is not growing (except for slight increases in suicide). The golden mean parabola, known since the times of the Egyptians and Babylonians, is the function that governs the human mind. It's what goes on inside the black box.

Keywords: psychology; psychiatry; cusack psychiatric hospitalization formula; at math; golden mean parabola; human mind

\section{Introduction}

After reading the DSM-5 for psychology diagnosis, I noticed that there is a lot of mental illness in our modern society. Is society getting more and more mentally ill over the centuries? I thought a good proxy would be psychiatric hospital patient populations. Since we have had the oldest psychiatric hospital in British North America, I looked up the data at the NB Museum. What I found was that there scant data from prior to the 1900 's, but there were causes of deaths over 25 years from 1787-1812. There were 2 suicides in that period. Not much to work with, but something. I continued and found The NB Provincial Hospital (Lunatic Asylum) admissions for 5 distinct years. The data is in the table below. From these data, I curve fitted an exponential function to the data and predicted that in 2020, there should have been 226 admissions to the current floor of the Saint John Regional Hospital. That is about right at 18.8 patients per month in a hospital that serves 200,000 population. Subsequently, I applied AT Math to the equations and data. And found that the hospitalizations follow the standard second order differential equation. If take the human mind to be governed by the golden mean parabola $\left(\mathrm{t}^{\wedge} 2-\mathrm{t}-1=0\right)$, the constants from AT Math drop out. What this all means is that psychiatric hospitalizations and suicides follow distinct physics. There is nothing random about it.

\begin{tabular}{|l|l|}
\hline X=Year & Y=Hospitalizations \\
\hline $1787-1812$ & 400 \\
\hline 1904 & 562 \\
\hline 1934 & 888 \\
\hline 1969 & 100 \\
\hline 1976 & 1697 \\
\hline 2020 PREDICTED & 226 \\
\hline
\end{tabular}

Table 1 Historical Hospitalizations to Psychiatric Hospitals

These data can be curve fitted to the following exponential function $(\mathrm{R}=0.8491)$

\section{Cusack Psychiatric Hospitalization Formula:}

$\mathrm{y}=440.6071$ - $(-6.422992 \mathrm{e}-25 /-0.02993871) *\left(1-\mathrm{e}^{\wedge}(+0.02993871 * \mathrm{x})\right)$

For 2020: Predicted 226 psychiatric hospitalizations for a regional

hospital serving 200,000 people $=226 / 200,000=113 / 100,000$

Suicides from $200-2015=14 / 100,000$

$14 / 113=12.38=1 / 0.807=1 / \mathrm{c}^{4}$

$0.807^{0.25}=2.99792458 \sim \mathrm{c}$

$\mathrm{P}=\mathrm{i}^{2} \mathrm{R}$

$1.77=\left(1 / \mathrm{c}^{2}\right)^{2} \mathrm{R}$

$\mathrm{R}=14.29=1 / 6.99429 \sim 1 / 7$ Economic Multiplier

$\mathrm{V}=\mathrm{iR}$

$105.5=\left(1 / c^{2}\right) \mathrm{R}$

$\mathrm{R}=1-5.5\left(\mathrm{c}^{2}\right)$

$=948$

$=1 / 105.5=1 / \mathrm{V}+$

$\mathrm{V}=\mathrm{iR}$

$\mathrm{V}=\mathrm{i}(1 / \mathrm{V})$

$\mathrm{V}^{2}=\mathrm{i}$

$\mathrm{I}=0.11130=1 / 2.997=\mathrm{c}$

$1 / 6.99 \times 0 / 807=115.15=1 / 0.866=\sin 60^{\circ}$

Second Order Differential Equation: 
$y^{\prime \prime}+y^{\prime}+y=0$

The function of the human mind is the golden mean parabola. Inputting,

$2+(2 \mathrm{t}-1)+\left(\mathrm{t}^{2}-\mathrm{t}-1\right)=0$

$\mathrm{t}^{2}-\mathrm{t}=0$

$\mathrm{t}=0 ; 1$

$\mathrm{t}=1 \quad \mathrm{E}=1 / \mathrm{t}=1$

$\mathrm{s}=\mathrm{t}$

$\mathrm{s}=\mathrm{E} \mathrm{xt}=|\mathrm{E}| \mathrm{t} \mid \sin \mathrm{t}$

$E=1 / \sin t$
$1=1 / \sin \mathrm{t}$

$\mathrm{t}=90^{\circ}=\pi / 2$

\section{How many people are affected by mental illness?}

792 million people are affected by mental health issues worldwide (2). At any given time, 1 in 6 working-age adults have symptoms associated with mental ill health (3). Mental illness is the second-largest source of burden of disease in England. Mental illnesses are more common, long-lasting and impactful than other health conditions $792,000,000 / 7,000,000,000=113.14$. This is as predicted by the Cusack Psychiatric Hospitalization Equation above.

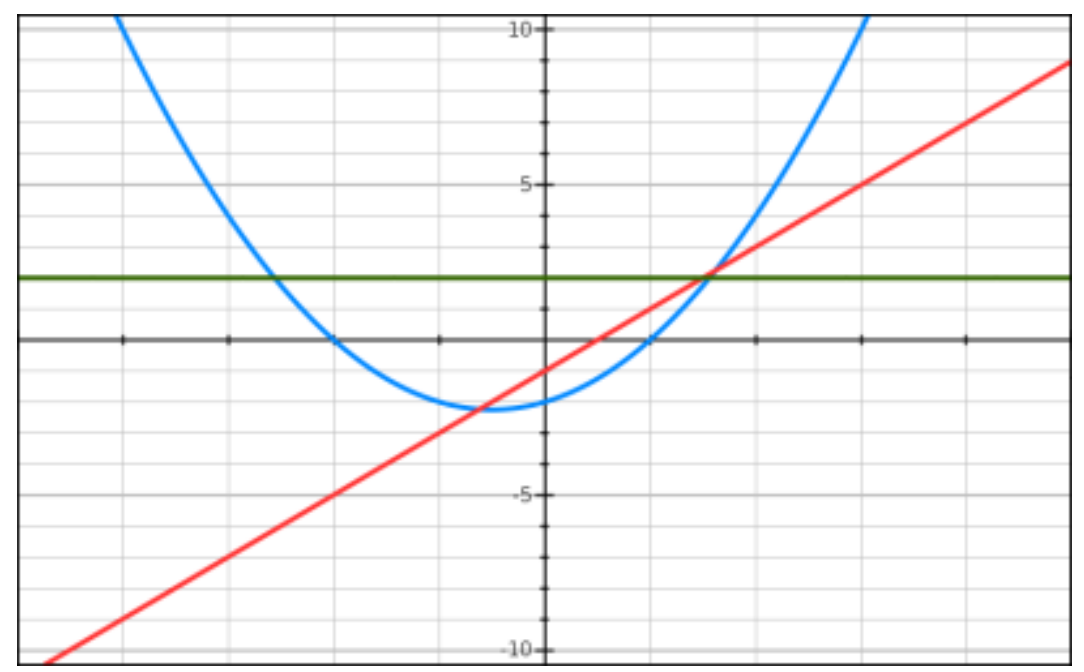

Figure $1 y^{\prime \prime}=2 ; y^{\prime}=2 t-1 ; y=t^{\wedge} 2-t-1$

One patient and his family suffer from several mental disorders including: Schizophrenia; Cyclothymic; Panic Disorders; Claustrophobia; Agoraphobia; Social anxiety; Male Hypoactive Sexual Disorder; Depersonalization Disorder; and Dependant Disorder, Dissociative Disorder and Self Destruction. Other closely related suffer from suffers from Hording Disorder; Schizotypal Disorder; Social anxiety Disorder, Negative mood. Another from Anorexia Nervosa; Dementia; Trauma. Another: Schizoid Personality disorder; Sleep-Wake Disorder. Finally, a Paranoid Personality Disorder. There is no shortage of mental illness in the world, but I conclude that it has not changed since humans became humans.

\section{Conclusion}

Since the function of the mind has not changed for thousands of years, then mental illness has changed either. The environment changes, but the function remains robust.

\section{References}

1. Hugh Hazen I=F90 Inquest into causes of death NB Museum S65 F90

2. Central Care Saint John., Inc. S156-28, F1; F1 Manuscript Provincial Hospital Saint John. NB Museum. 